\title{
Response of Gaussian-modulated Guided Wave in Aluminum: An Analytical, Numerical and Experimental Study
}

\author{
Sohaib Z Khan ${ }^{1, *}$, Muhammad A Khan ${ }^{1}$, Muhammad Tariq ${ }^{1}$, Kamran A Khan², \\ Tariq M Khan ${ }^{3}$, Taha $\mathrm{Ali}^{3}$ \\ ${ }^{1}$ Department of Engineering Sciences, PN Engineering College, National University \\ of Sciences and Technology, Karachi, Pakistan \\ ${ }^{2}$ Department of Aerospace Engineering, Khalifa University of Science, Technology \\ and Research (KUSTAR), Abu Dhabi, UAE \\ ${ }^{3}$ Department of Electrical and Power Engineering, PN Engineering College, National \\ University of Sciences and Technology, Karachi, Pakistan
}

* Corresponding author:

\section{Dr Sohaib Zia Khan}

Room 105, PG Building, PN Engineering Department of Engineering Sciences, PN Engineering College, National University of Sciences and Technology, Karachi, Pakistan

Email: consultation.drsohaib@gmail.com / Sohaib.Khan@pnec.nust.edu.pk

Phone: +923332273100 


\title{
Response of Gaussian-modulated Guided Wave in Aluminum: An Analytical, Numerical and Experimental Study
}

\begin{abstract}
:
The application of guided-wave ultrasonic testing in structural health monitoring has been widely accepted. Comprehensive experimental works have been performed in the past but their validation with possible analytical and numerical solutions still requires serious efforts. In this paper, behavior and detection of the Gaussian-modulated sinusoidal guided-wave pulse travelling in an aluminum plate are presented. An analytical solution is derived for sensing guided-wave at a given distance from the actuator. This solution can predict the primary wave modes separately. Numerical analysis is also carried out in COMSOL® Multiphysics software. An experimental setup comprising piezoelectric transducers is used for the validation. Comparison of experimental results with those obtained from analytical and numerical solutions shows close agreement.
\end{abstract}

\section{Introduction:}

Guided-waves have been used in ultrasonic testing mainly as a non-destructive testing (NDT) of plates and other structures [1]. These waves travel in a plate in the form of longitudinal and transverse components [2]. The major barrier in using guided waves for NDT is the formation of the multiple modes and their dispersion. There are several ways to avoid this, mainly by changing incident pulse frequency, pulse shape, transducer dimensions and sensor distance [3]-[5] as well as dispersion compensation procedure and other post signal processing techniques [6]. Convectional ultrasonic probes are heavy and expensive [7]. In addition, conventional methods are limited to test the vicinity beneath or close the transducer [8]. 
Whereas guided-waves can travel to large distances and offer advantages of inspecting large structures by using a minimum number of transducers [9]-[18]. Guided-waves move along the geometry, therefore, it has become very effective technique for the inspection of long length structures like pipelines and rails [19] and also it can be used by non-contact probing techniques [20]. In guided-waves, Piezoelectric Wafer Active Sensor (PWAS) is used that can be classified based on its functionality as: emitter / actuator, or receiver / sensor, or both. Single or multiple PWAS can be used in experimental testing. PWAS devices are light weight, inexpensive, non-invasive and can be used as both active and passive probes.

The effectiveness of guided-waves in structural health monitoring has been empirically and analytically studied on geometries like plates [7], annular structures [21], hollow thick cylinders [22] and for layered structures, based on global matrix method [23]. Most of the analytical research works are dealt with the emission and propagation of the waves only [2], [24]. Analytical models of standard waves, for example, sine waves, travelling in an elastic solid medium are available [2]. In experimental testing, modulated pulses are preferred [25]. However, rectangular or square pulses as the modulating signals are not feasible because they can excite larger spectrum and create additional modes which are difficult for the signal processing. To overcome the gradual mechanical response of the transducers, Gaussianmodulated waves are used by researchers [26]. However, an analytical solution for a Gaussian-modulated sinusoidal wave is not available.

The complexities involved in the analytical solutions have been diverting the attention of the researchers towards the numerical analysis of the guided-wave propagation. With the availability of many commercial software, numerical analysis helped in dealing with the complex geometries and defects in materials [7], [8], [27]-[29]. Despite of advancement, numerical analysis requires analytical or experimental validation. 
In this paper, behavior and detection of the Gaussian-modulated sinusoidal guided-wave on an aluminum plate is presented. First, the analytical solution is derived, which is capable of sensing guided-wave at a given distance from the actuator. The solution can predict primary wave modes individually. A numerical simulation is also carried out in COMSOL® Multiphysics software. In the last section, experimental results are presented, and comparison with the analytical and numerical solutions is discussed.

\section{Materials and Methods:}

Aluminum alloy 6061-T6 was used in experimentation. The dimensions of the specimen were

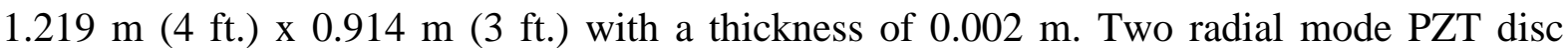
wafer transducers made up of P-33 piezo-ceramic material (equivalent to PZT-5A) were used. One of the transducers was used as an actuator and the other as a sensor. Both the actuator and the sensor were coupled using cyanoacrylate. A rigid bond was produced between the plate and the transducers. Both transducers were placed on the middle of the plate to avoid reflected waves and the distance between the transducers was considered as $x$, as shown in Figure 1. The diameter and the thickness of the transducers were $10 \mathrm{~mm}$ and 1 $\mathrm{mm}$, respectively. The selected transducers have a resonant frequency of $200 \mathrm{kHz}$. Thus, only primary anti-symmetric $\left(A_{0}\right)$ and symmetric $\left(S_{0}\right)$ modes were generated and the higher modes were avoided to simplify signal processing. The secondary modes for aluminum are excited above $1000 \mathrm{kHz} \cdot \mathrm{mm}$, which was higher than the setup used in this study. The shear wave velocity $\left(C_{s}\right)$ for aluminum is $3111 \mathrm{~m} / \mathrm{s}$ [30]. The phase velocities $C^{\mathrm{So}}$ for $\mathrm{S}_{0}$ and $\mathrm{C}^{\mathrm{Ao}}$ for $\mathrm{A}_{0}$ are found to be $5650 \mathrm{~m} / \mathrm{s}$ and $1755 \mathrm{~m} / \mathrm{s}$ whereas group velocities $\mathrm{C}_{\mathrm{g}}$ So for $\mathrm{S}_{0}$ and $\mathrm{C}_{\mathrm{g}}{ }^{\text {Ao }}$ for $\mathrm{A}_{0}$ are found to be $5300 \mathrm{~m} / \mathrm{s}$ and $2600 \mathrm{~m} / \mathrm{s}$, respectively [2]. 
A Gaussian-modulated pulse was used for the excitation. The signal was amplitude modulated over the resonant frequency of the PZT transducer for efficient conversion into a mechanical domain. The Gaussian parameters were: mean $\left(\mathrm{t}_{\mathrm{m}}\right)=1.13 \times 10^{-5} \mathrm{~s}$ and standard deviation $\left(\mathrm{t}_{0}\right)=4.5 \times 10^{-6} \mathrm{~s}$. An Arbitrary Function Generator (AFG), GW-Instek AFG-2005, was used to generate a pulse to excite the actuator as shown in Figure 2. The response signal from the sensor was analyzed with a digital storage oscilloscope (GW-Instek GDS-1000).

\section{Analytical Study and Numerical Solution:}

\subsection{Analytical Study:}

The actuator was excited by five counts of Gaussian modulated tone burst. This was obtained by multiplying the sine wave at a frequency $(f)$ of $200 \mathrm{kHz}$ with the Gaussian function, as shown in Figure 2. The input signal is shown in Eq 1. In this equation, $\omega=2 \pi f$ is the natural frequency, $t$ is the time at any instance, $t_{m}$ and $t_{o}$ dictates the mean and the standard deviation of the Gaussian function, respectively. For brevity and quick referencing all the symbols are also summarized in Table 1.

$$
x(t)=e^{-0.5\left(\frac{t-t_{m}}{t_{o}}\right)^{2}} \sin (\omega t)
$$

Guided wave propagation takes place along $\mathrm{X}$-axis whereas it has a particle motion in the $\mathrm{XZ}$ plane as shown in Figure 3. The particle motion $U_{z}$ is given by Eq 2. In this equation, $h(z)$ is the standing wave across the plate thickness and $\xi$ is the wave number.

$$
U_{z}(x, z, t)=[h(z)] \cdot\left[e^{-0.5\left(\frac{t-t_{m}}{t_{o}}\right)^{2}} \sin (\omega t-\xi x)\right] \quad \text { Eq. } 2
$$

The wave equation is given by [2] as shown in Eq. 3 .

$$
\nabla^{2} U_{z}=\frac{1}{C_{s}^{2}} \ddot{U}_{z}
$$


Where, $\nabla^{2}$ is the Laplace operator, $C_{S}$ is the shear wave velocity and $\ddot{U}_{z}$ is the double derivative of $U_{z}$ with respect to time.

$$
\left(\frac{\partial^{2}}{\partial x^{2}}+\frac{\partial^{2}}{\partial z^{2}}\right) \cdot U_{z}=\frac{1}{C_{s}^{2}} \ddot{U}_{z}
$$

Eq. 4

Assuming Y invariant and differentiating each term separately from the Eq. 4 with respect to their respective variables, $x, z, t$ are shown for brevity in Eq. 5.1, Eq. 5.2 and Eq. 5.3, respectively.

$$
\begin{array}{cc}
\frac{\partial^{2} U_{z}}{\partial x^{2}}=\left(-\xi^{2}\right) h(z) e^{-0.5\left(\frac{t-t_{m}}{t_{o}}\right)^{2}} \sin (\omega t-\xi x) & \text { Eq. } 5.1 \\
\frac{\partial^{2} U_{z}}{\partial z^{2}}=h^{\prime \prime}(z) e^{-0.5\left(\frac{t-t_{m}}{t_{o}}\right)^{2}} \sin (\omega t-\xi x) & \text { Eq. } 5.2 \\
\ddot{U}_{z}=h(z) e^{-0.5\left(\frac{t-t_{m}}{t_{o}}\right)^{2}} \sin (\omega t-\xi x) \cdot\left[-\omega^{2}-\frac{1}{t_{o}{ }^{2}}-2 \omega\left(\frac{t-t_{m}}{t_{o}{ }^{2}}\right) \cot (\omega t-\xi x)+\right. \\
\left.\left(\frac{t-t_{m}}{t_{o}{ }^{2}}\right)^{2}\right] \text { Eq. } 5.3
\end{array}
$$

Putting the values of Eq. 5.1, 5.2 and 5.3 in Eq. 4 and after simplification it is shown in Eq. 6,

$$
\left(-\xi^{2}\right) h(z)+h^{\prime \prime}(z)=\frac{1}{c_{s}{ }^{2}} h(z)\left[-\omega^{2}-\frac{1}{t_{o}{ }^{2}}-2 \omega\left(\frac{t-t_{m}}{t_{o}{ }^{2}}\right) \cot (\omega t-\xi x)+\left(\frac{t-t_{m}}{t_{o}{ }^{2}}\right)^{2}\right]
$$

Eq. 6

For the purpose of simplification, a new variable $\eta$ is introduced as shown in Eq. 7.

$$
\eta^{2}=\frac{1}{C_{s}{ }^{2}}\left[\omega^{2}+\frac{1}{t_{o}^{2}}+2 \omega\left(\frac{t-t_{m}}{t_{o}{ }^{2}}\right) \cot (\omega t-\xi x)-\left(\frac{t-t_{m}}{t_{o}^{2}}\right)^{2}\right]-\xi^{2}
$$

By substituting Eq. 7 into Eq. 6, it will become a second order differential equation as shown in Eq. 8. The general solution of this second order differential equation is shown in Eq. 9. 


$$
\begin{gathered}
h^{\prime \prime}(z)+\eta^{2} h(z)=0 \\
h(z)=C_{1} \sin \eta z+C_{2} \cos \eta z
\end{gathered}
$$

Eq. 8

Eq. 9

By substituting Eq. 9 back into the Eq. 2 will give Eq. 10.

$$
U_{z}(x, z, t)=\left(C_{1} \sin \eta z+C_{2} \cos \eta z\right) e^{-0.5\left(\frac{t-t_{m}}{t_{o}}\right)^{2}} \sin (\omega t-\xi x) \quad \text { Eq. } 10
$$

The solution of the above equation results in trivial form. However, the constants can be determined by separating Eq 10 it into symmetric and anti-symmetric modes as performed in [2], [31].

\section{Symmetric mode}

For the symmetric mode, using the following boundary condition,

$$
\left.U_{z}(x, z, t)\right|_{z=d}=-\left.U_{z}(x, z, t)\right|_{z=-d}
$$

Putting this boundary condition in Eq. 10,

$$
\begin{aligned}
\left(C_{1} \sin \eta d+\right. & \left.C_{2} \cos \eta d\right) e^{-0.5\left(\frac{t-t_{m}}{t_{o}}\right)^{2}} \sin (\omega t-\xi x) \\
& =\left(C_{1} \sin (-\eta d)+C_{2} \cos (-\eta d)\right) e^{-0.5\left(\frac{t-t_{m}}{t_{o}}\right)^{2}} \sin (\omega t-\xi x)
\end{aligned}
$$

Above boundary condition is only satisfied when,

$$
\cos \eta d=0
$$

Then $\eta^{s} d$ for the symmetric mode will be

$$
\begin{aligned}
\eta^{s} d=\frac{\pi}{2}, 3 \frac{\pi}{2}, 5 \frac{\pi}{2}, \ldots,(2 n+1) \frac{\pi}{2}, & \mathrm{n}=0,1,2 \ldots \\
\cos \eta^{s} d=0 \quad \text { and } & \sin \eta^{s} d= \pm 1
\end{aligned}
$$


Putting these in Eq. 10 will become,

$$
U_{z}^{s}(x, z, t)=C_{1} e^{-0.5\left(\frac{t-t_{m}}{t_{o}}\right)^{2}} \sin (\omega t-\xi x)
$$

The value of $C_{1}$ acts as a scaling factor and can be used for the amplification a signal. Therefore, it can be ignored for wave pattern analyses if the signals are normalized with their peak values. Eq. 11 presents a symmetric wave pattern received at the sensor. In this equation, the travelling time required by the wave to reach the sensor from the actuator is dependent on the wave mode velocity. The signal may experience amplitude loss and dispersion during propagation, which has been ignored because of the small distance between the two transducers. The sensor will respond once the wave reaches the sensor. The variable $t$ is replaced by $t-x / C_{g} s_{0}$ to accommodate the effect of distance, where $x$ is the distance between the both transducers and $C_{g} S_{0}$ is the $S_{0}$ group velocity.

\section{Anti-symmetric mode}

Similar procedure is adopted for the antisymmetric mode, using following boundary condition,

$$
\left.U_{z}(x, z, t)\right|_{z=d}=\left.U_{z}(x, z, t)\right|_{z=-d}
$$

Putting this boundary condition in Eq. 10,

$$
\begin{aligned}
& \left(C_{1} \sin \eta d+C_{2} \cos \eta d\right) e^{-0.5\left(\frac{t-t_{m}}{t_{o}}\right)^{2}} \sin (\omega t-\xi x) \\
& \quad=\left(C_{1} \sin (-\eta d)+C_{2} \cos (-\eta d)\right) e^{-0.5\left(\frac{t-t_{m}}{t_{o}}\right)^{2}} \sin (\omega t-\xi x)
\end{aligned}
$$

This leads to $\sin \eta d=0$. Then $\eta^{A} d$ that is for the antisymmetric mode will be

$$
\eta^{A} d=0, \pi, 2 \pi, \ldots, n \pi, \quad \mathrm{n}=0,1,2 \ldots
$$




$$
\sin \eta^{A} d=0 \quad \text { and } \quad \cos \eta^{A} d= \pm 1
$$

Substituting these values in Eq .10,

$$
U_{z}^{A}(x, z, t)=C_{2} e^{-0.5\left(\frac{t-t_{m}}{t_{o}}\right)^{2}} \sin (\omega t-\xi x)
$$

Again $C_{2}$ is only a scaling factor. Similar to the Eq. 11, travelling time $t$ in the Eq. 12 is replaced by $-x / C_{g}{ }^{A_{0}}$, where $C_{g}{ }^{A_{0}}$ is the $A_{0}$ group wave velocity.

Using Eq. 11 and Eq. 12, both modes are plotted separately, as shown in Figure 4, for two distances between the transducers: (a) $50 \mathrm{~mm}$ and (b) $100 \mathrm{~mm}$. Both modes can be observed separating from each other due to the difference in their propagation speed. This also shows how both modes will be distinctively received at the sensor, which is not possible in experiments. Figure 5 shows the combined output waveform for various distances between the two transducers received at the sensor. In Figure 4(a), the distance is only $50 \mathrm{~mm}$ and the travelling time is not long enough for the complete separation of $S_{0}$ and $A_{0}$ modes. Therefore, fourth peak of $S_{0}$ waveform is superimposed by the second peak of $A_{0}$ waveform. This superimposed amplitude can be noticed in combined waveform (Figure 5(a)). In the case, when the distance between the transducers is $100 \mathrm{~mm}$, these modes are well separated. 


\subsection{Numerical Solution:}

COMSOL® Multiphysics software was used for a numerical solution of the described setup.

A 2D model of the experimental setup was defined having a cross-section of both plate and transducers. The propagation of guided waves is axis-symmetric and can be easily represented by a $2 \mathrm{D}$ numerical model. All the dimensions were the same as defined above, except the transducers' diameter because they are not in complete contact with the plate (see inset of Figure 1). The effective diameter of the imprint area of the transducers was taken to be $7 \mathrm{~mm}$ instead of $10 \mathrm{~mm}$ and hence compensation was assumed.

In the software, the 2D-Piezoelectric Devices (PZD) domain was selected for the model. Material for the plate and the transducers was selected from the software library as 'aluminum alloy 6061 (UNS A96061)-[Solid,-T6]' and 'Lead Zirconate Titanate (PZT-5A)', respectively. A very fine triangular element mesh was selected for the model. The maximum and the minimum element size was $15 \mathrm{~mm}$ and $0.02 \mathrm{~mm}$, respectively. Values of maximum element growth rate, resolution of curvature and resolution of the narrow region were taken 1.05, 0.15 , and 1 , respectively. The model had a mesh consisting of 2175 domain elements and 1621 boundary elements.

The boundary conditions shown in Figure 6, were defined as follows. Edges 'A' were considered as zero charge boundaries. Edges 'B' were defined as grounded. The electric potential was applied on the top edge ' $\mathrm{C}$ ' of the actuator. The meshing of the assembly was adopted in such a way that high density mesh regions were defined near the contact points between the both transducers and the plate to capture better resolution of the analysis, as shown in Figure 7. The dimension of the plate was much bigger than the transducers and thus, away from the contact points, low density mesh was adopted. This reduced overall number of mesh in the assembly model. A very fine triangular element mesh was selected for 
the model. The maximum and the minimum element size was $15 \mathrm{~mm}$ and $0.02 \mathrm{~mm}$, respectively. Values of maximum element growth rate, resolution of curvature and resolution of the narrow region were taken $1.05,0.15$, and 1 , respectively. The model had a mesh consisting of 2175 domain elements and 1621 boundary elements. Time dependent study with step size of $0.1 \mu$ s was selected with relative tolerance of 0.01 . Simulation was continued until the sensor received one complete pulse emitted by the actuator. Figure 8 shows the results obtained after post-processing.

\section{Experimental Results and Discussion:}

The experiments were performed for two distances between the transducers: $50 \mathrm{~mm}$ and 100 $\mathrm{mm}$. The received signals at the sensor obtained on the oscilloscope were similar as predicted by the analytical and the numerical analysis. For the comparison purpose, data obtained by analytical, numerical and experimental observations are shown together in Figure 9. No filtering was applied on the experimental data. The transducers were placed far away from the edges of the aluminum plate $(>50 \mathrm{~cm})$ which ultimately delayed the reflected pulse from the edges by an adequate long time, avoiding interference with the first received pulse. The first reflection was expected to reach back to the sensor approximately at $170 \mu$ s. Signals received experimentally at the sensor had both $A_{0}$ and $S_{0}$ modes. $S_{0}$ is non-dispersive, but $A_{0}$ is dispersive in nature. A careful observation revealed that once the slower and dispersive mode $\left(A_{0}\right)$ reached the sensor, the signals slightly started deviating from the predicted pattern. It has been reported in the literature that the results obtained for different transducer pairs were quite similar for the $S_{0}$ mode in both amplitude and frequency dependence, while the $A_{0}$ results were significantly different [28]. The principle reason reported in that paper was a poor bonding with the plate. In the current study, the connection between the plate and the transducers was not ideal despite a rigid bond was created by using cyanoacrylate as a 
couplant. This deviation may also be explained by the behavior of the actuator. During experimentation, it was found that the actuator kept on vibrating at the smaller amplitude even after the input pulse was delivered as shown in Figure 10. This resulted in unwanted noise in the input signals, which was unfortunately unavoidable in the current set-up.

\section{Conclusion:}

Detection and behavior of the Gaussian-modulated guided wave pulse travelling in an aluminum plate is presented. Experiments were performed by applying Gaussian modulated sinusoidal pulse as an input signal on the actuator and the results were collected on the sensor through an oscilloscope. The analytical solution is also derived by solving the general wave equation for the symmetric and the anti-symmetric modes, which not only explained the formation and propagation of both fundamental modes but also their superposition and separation. Moreover, the analytical model is capable of producing separate formation of both the modes at the sensor. Numerical modelling of GWUT was done by using COMSOL® Multiphysic and boundary conditions were applied similar to the experimental setup. The results of the response of the Gaussian-modulated guided-wave in aluminum plate using analytical, numerical and experimental study show close agreement. The method can be extended for detecting defects in material.

\section{References:}

[1] A. Raghavan and C. E. Cesnik, "Review of guided-wave structural health monitoring," Shock Vib. Dig., vol. 39, no. 2, pp. 91-116, 2007.

[2] V. Giurgiutiu, Structural health monitoring with piezoelectrical wafer active sensors. Academic Press, 2007.

[3] F. De Villa, E. Roldan, C. Tirado, R. Mares, S. Nazarian, and R. A. Osegueda, "Defect Detection in thin plates using So Lamb wave scanning," presented at the 6th Annual 
International Symposium on NDE for Health Monitoring and Diagnostics, 2001, pp. 121-130.

[4] M. J. S. Lowe, D. N. Alleyne, and P. Cawley, "The Mode Conversion of a Guided Wave by a Part-Circumferential Notch in a Pipe," J. Appl. Mech., vol. 65, no. 3, pp. 649-656, Sep. 1998.

[5] S. S. Kessler, S. M. Spearing, and M. J. Atalla, "In-situ damage detection of composites structures using Lamb wave methods," in Proceedings of the First European Workshop on Structural Health Monitoring, 2002, pp. 10-12.

[6] L. De Marchi, A. Marzani, and M. Miniaci, "A dispersion compensation procedure to extend pulse-echo defects location to irregular waveguides," NDT E Int., vol. 54, pp. 115-122, Mar. 2013.

[7] V. Giurgiutiu, J. Bao, and W. Zhao, "Piezoelectric wafer active sensor embedded ultrasonics in beams and plates," Exp. Mech., vol. 43, no. 4, pp. 428-449, Dec. 2003.

[8] F. Moser, L. J. Jacobs, and J. Qu, "Modeling elastic wave propagation in waveguides with the finite element method," Ndt E Int., vol. 32, no. 4, pp. 225-234, 1999.

[9] P. Cawley, M. J. S. Lowe, D. N. Alleyne, B. Pavlakovic, and P. Wilcox, "Practical long range guided wave inspection-applications to pipes and rail," Mater. Eval., vol. 61, no. 1, pp. 66-74, 2003.

[10] P. Cawley and D. Alleyne, "The use of Lamb waves for the long range inspection of large structures," Proc. Ultrason. Int. 1995, vol. 34, no. 2-5, pp. 287-290, Jun. 1996.

[11] T. R. Hay and J. L. Rose, "Flexible PVDF comb transducers for excitation of axisymmetric guided waves in pipe," Sens. Actuators Phys., vol. 100, no. 1, pp. 18-23, Aug. 2002.

[12] M. J. S. Lowe, D. N. Alleyne, and P. Cawley, "Defect detection in pipes using guided waves," Ultrason. Int. 1997, vol. 36, no. 1-5, pp. 147-154, Feb. 1998.

[13] J. Mu, L. Zhang, and J. L. Rose, "Defect circumferential sizing by using long range ultrasonic guided wave focusing techniques in pipe," Nondestruct. Test. Eval., vol. 22, no. 4, pp. 239-253, Oct. 2007.

[14] J. Pei, M. I. Yousuf, F. L. Degertekin, B. V. Honein, and B. T. Khuri-Yakub, "Lamb wave tomography and its application in pipe erosion/corrosion monitoring," Res. Nondestruct. Eval., vol. 8, no. 4, pp. 189-197, Dec. 1996.

[15] J. L. Rose, Ultrasonic waves in solid media. Cambridge [u.a.]: Cambridge University Press, 1999.

[16] I. A. Viktorov, Rayleigh and Lamb waves: physical theory and applications. New York: Plenum Press, 1967.

[17] F. Lanza di Scalea, P. Rizzo, and A. Marzani, "Propagation of ultrasonic guided waves in lap-shear adhesive joints: Case of incident a0 Lamb wave," J. Acoust. Soc. Am., vol. 115, no. 1, pp. 146-156, 2004.

[18] F. Lanza di Scalea, H. Matt, and I. Bartoli, "The response of rectangular piezoelectric sensors to Rayleigh and Lamb ultrasonic waves," J. Acoust. Soc. Am., vol. 121, no. 1, pp. 175-187, 2007. 
[19] P. Rizzo, M. Cammarata, I. Bartoli, F. L. di Scalea, S. Salamone, S. Coccia, and R. Phillips, "Ultrasonic Guided Waves-Based Monitoring of Rail Head: Laboratory and Field Tests," Adv. Civ. Eng., vol. 2010, p. 13, 2010.

[20] S. Coccia, R. Phillips, I. Bartoli, S. Salamone, F. di Scalea, M. Fateh, and G. Carr, "Noncontact Ultrasonic Guided-Wave System for Rail Inspection," Transp. Res. Rec. J. Transp. Res. Board, vol. 2261, pp. 143-147, Dec. 2011.

[21] G. Liu and J. Qu, "Transient wave propagation in a circular annulus subjected to transient excitation on its outer surface," J. Acoust. Soc. Am., vol. 104, no. 3, pp. 12101220, Sep. 1998.

[22] A. Ziaja, L. Cheng, Z. Su, P. Packo, L. Pieczonka, T. Uhl, and W. Staszewski, "Thick hollow cylindrical waveguides: A theoretical, numerical and experimental study," $J$. Sound Vib., vol. 350, pp. 73-90, Aug. 2015.

[23] M. D. Beard and M. J. S. Lowe, "Non-destructive testing of rock bolts using guided ultrasonic waves,” Int. J. Rock Mech. Min. Sci., vol. 40, no. 4, pp. 527-536, Jun. 2003.

[24] Victor Giurgiutiu, "Lamb Wave Generation with Piezoelectric Wafer Active Sensors for Structural Health Monitoring," in SPIE's 10th Annual International Symposium on Smart Structures and Materials and 8th Annual International Symposium on NDE for Health Monitoring and Diagnostics, 2-6 March 2002, San Diego, CA. paper \# 5056-17, 2002.

[25] W. J. Staszewski, B. C. Lee, L. Mallet, and F. Scarpa, "Structural health monitoring using scanning laser vibrometry: I. Lamb wave sensing," Smart Mater. Struct., vol. 13, no. 2, p. 251, 2004.

[26] Z. Hai-Yan and Y. Jian-Bo, "Piezoelectric transducer parameter selection for exciting a single mode from multiple modes of Lamb waves," Chin. Phys. B, vol. 20, no. 9, p. 094301, 2011.

[27] P. Frank Pai, H. Deng, and M. J. Sundaresan, "Time-frequency characterization of lamb waves for material evaluation and damage inspection of plates," Mech. Syst. Signal Process., vol. 62-63, pp. 183-206, Oct. 2015.

[28] J. N. J.H. Nieuwenhuis and I. J. O. D.W. Greve, "Generation and detection of guided waves using PZT wafer transducers," Ultrason. Ferroelectr. Freq. Control IEEE Trans. On, vol. 52, no. 11, pp. 2103 - 2111, Nov. 2005.

[29] R. Raišutis, R. Kažys, E. Žukauskas, L. Mažeika, and A. Vladišauskas, “Application of ultrasonic guided waves for non-destructive testing of defective CFRP rods with multiple delaminations," NDT E Int., vol. 43, no. 5, pp. 416-424, Jul. 2010.

[30] M. de Podesta, Understanding the properties of matter, 2nd ed. Taylor and Francis, 2002.

[31] V. Giurgiutiu and A. N. Zagrai, "Characterization of piezoelectric wafer active sensors," J. Intell. Mater. Syst. Struct., vol. 11, no. 12, pp. 959-976, 2000. 
Tables:

Table 1 : Abbreviation of symbols used in derivation.

\begin{tabular}{|c|l|}
\hline Abbreviation & \multicolumn{1}{|c|}{ Description } \\
\hline AFG & Arbitrary Function Generator \\
\hline $\mathrm{A}_{\mathrm{o}}$ & Primary Antisymmetric mode \\
\hline $\mathrm{C}^{\text {Ao }}$ & Phase velocity of $\mathrm{A}_{\mathrm{o}}$ mode \\
\hline $\mathrm{C}_{\mathrm{g}}{ }^{\text {ao }}$ & Group velocity of $\mathrm{A}_{\mathrm{o}}$ mode \\
\hline $\mathrm{C}_{\mathrm{g}}$ So & Group velocity of $\mathrm{S}_{\mathrm{o}}$ mode \\
\hline $\mathrm{C}_{\mathrm{s}}$ & Shear wave velocity \\
\hline $\mathrm{C}^{\mathrm{So}}$ & Phase velocity of $\mathrm{S}_{\mathrm{o}}$ mode \\
\hline $\mathrm{NDT}_{\mathrm{PWAS}}$ & Non-destructive testing \\
\hline $\mathrm{PZT}^{\mathrm{T}}$ & Piezoelectric Wafer Active Sensor \\
\hline $\mathrm{S}_{\mathrm{o}}$ & Lead Zirconate Titanate \\
\hline $\mathrm{t}_{\mathrm{m}}$ & Primary Symmetric mode \\
\hline $\mathrm{t}_{\mathrm{o}}$ & Mean of Gaussian function \\
\hline $\mathrm{U}_{\mathrm{z}}$ & Standard deviation of Gaussian function \\
\hline$\zeta$ & Particle motion in z-axis \\
\hline$\omega$ & Wave number \\
\hline & Angular velocity \\
\hline
\end{tabular}


Figures:

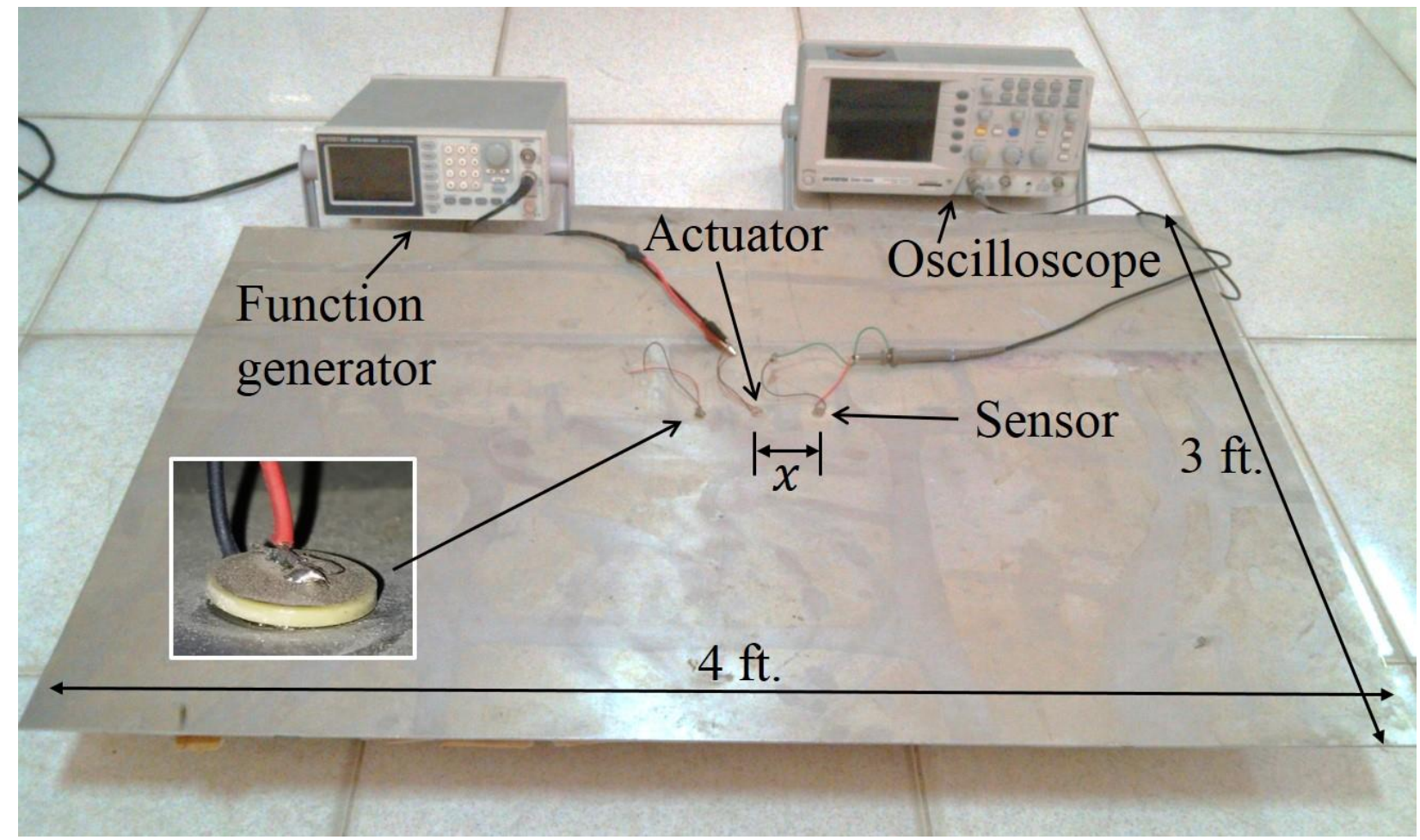

Figure 1: An illustration of the experimental setup. Inset showing the coupling of the transducer with the plate.

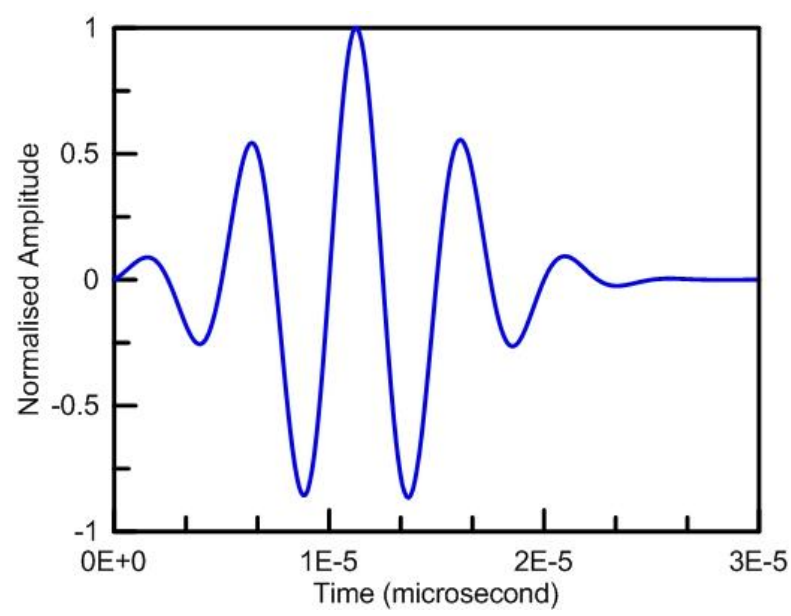

Figure 2: A Gaussian-modulated sinusoidal excitation pulse with $200 \mathrm{kHz}$ 5-counts Gaussian tone burst. 


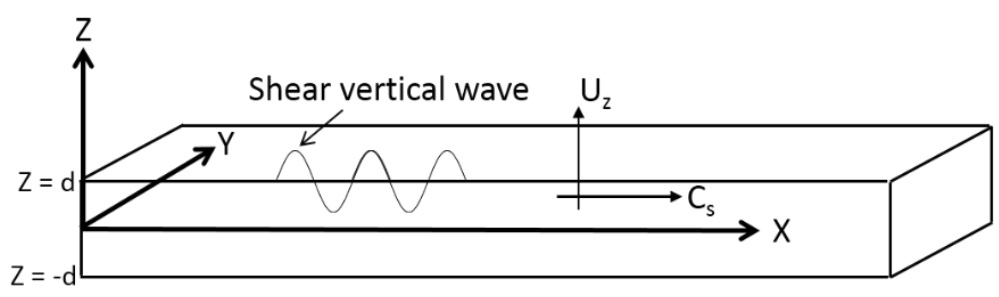

Figure 3: The propagation of shear vertical wave.
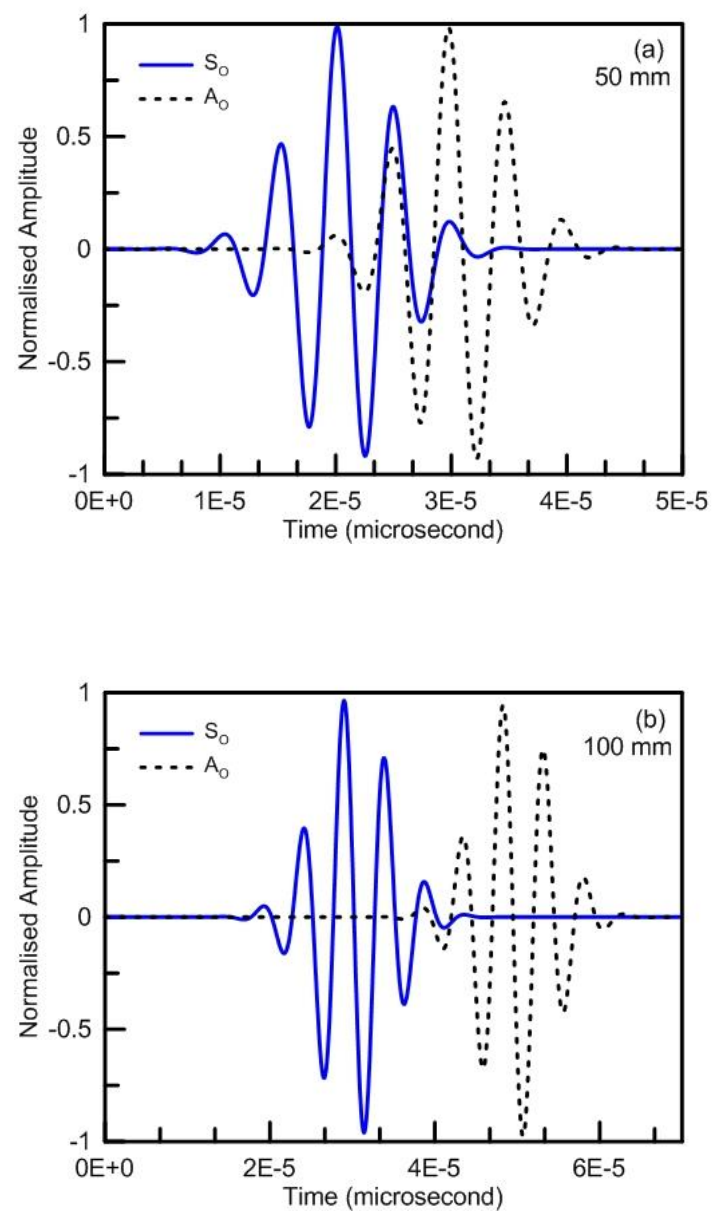

Figure 4: The symmetric and anti-symmetric mode of the guided wave received at the sensor calculated by the analytical solution at the distance between the actuator and sensor of (a) 50 $\mathrm{mm}$ and (b) $100 \mathrm{~mm}$. 

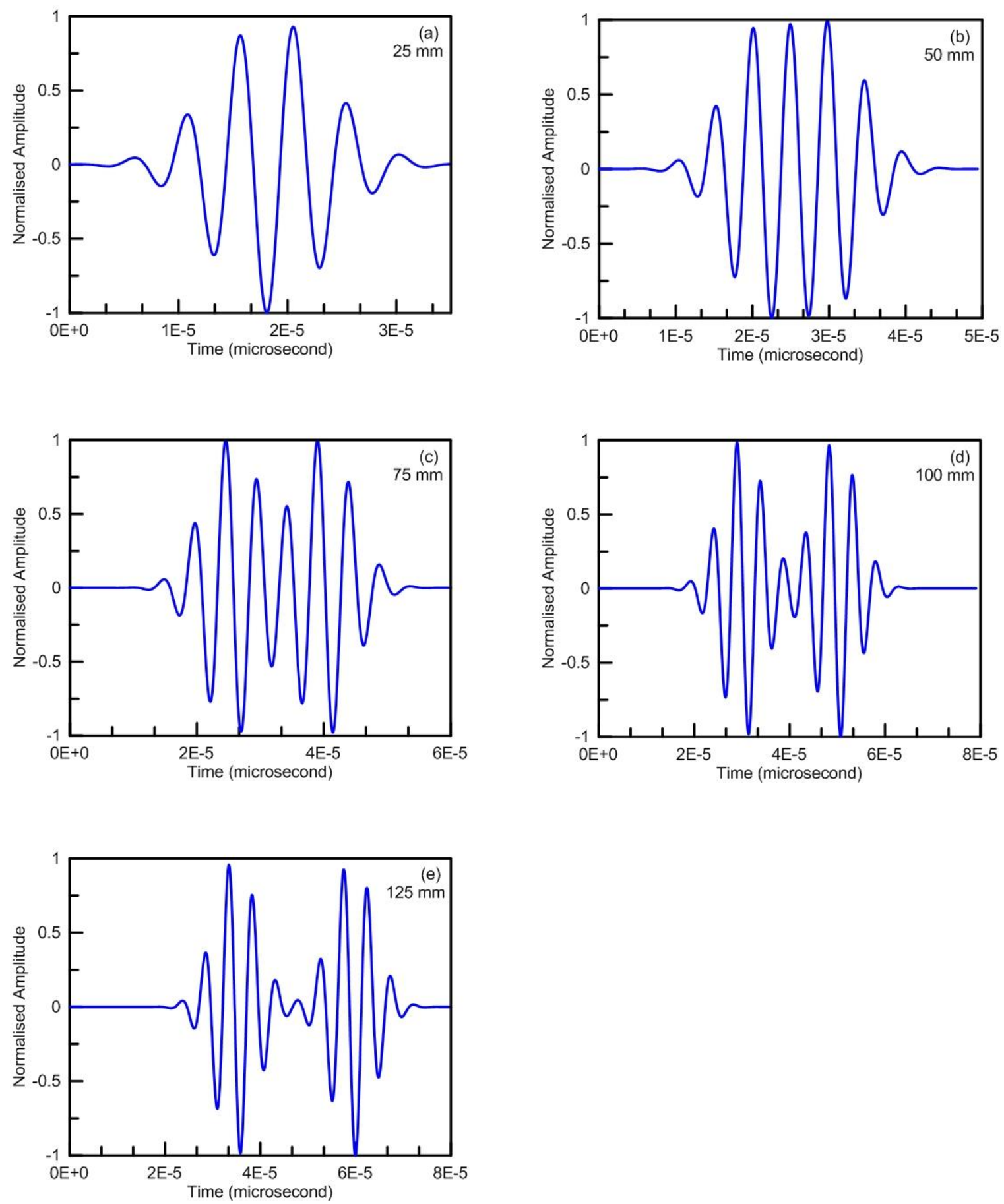

Figure 5: Guided-wave received at the sensor calculated by the analytical solution for the distances between the actuator and sensor of: (a) $25 \mathrm{~mm}$, (b) $50 \mathrm{~mm}$, (c) $75 \mathrm{~mm}$, (d) $100 \mathrm{~mm}$ and (e) $125 \mathrm{~mm}$. 


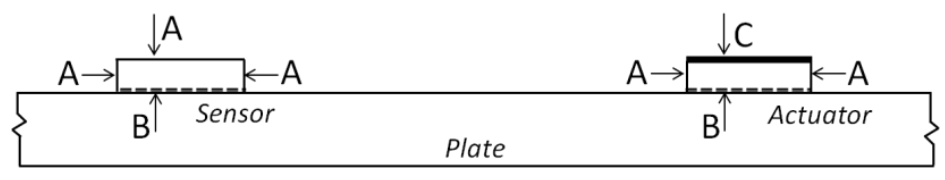

Figure 6: Boundary conditions set for the numerical analysis. 


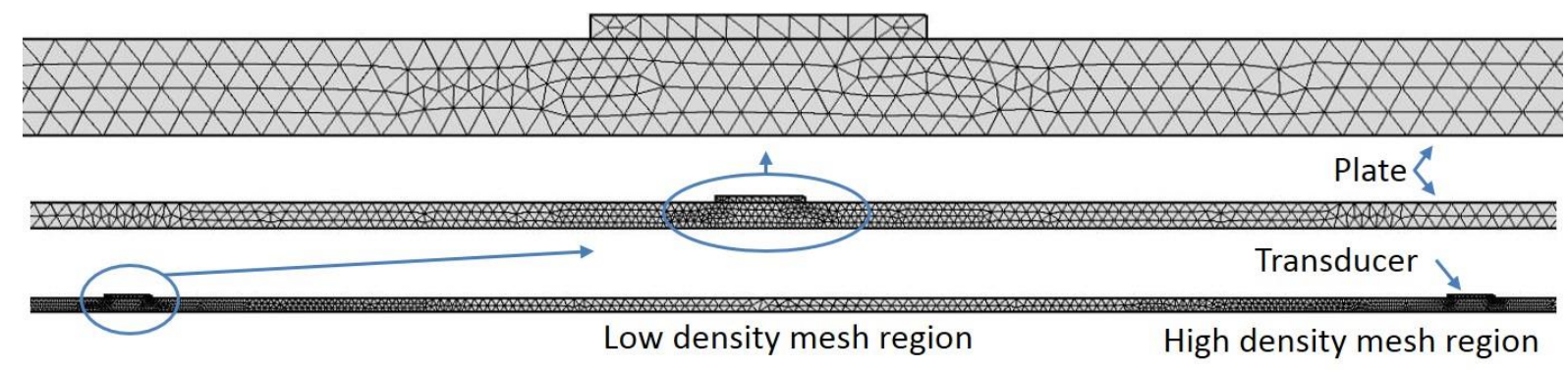

Figure 7: Mesh model of the assembly showing high and low density mesh regions around and away from the both transducers. 

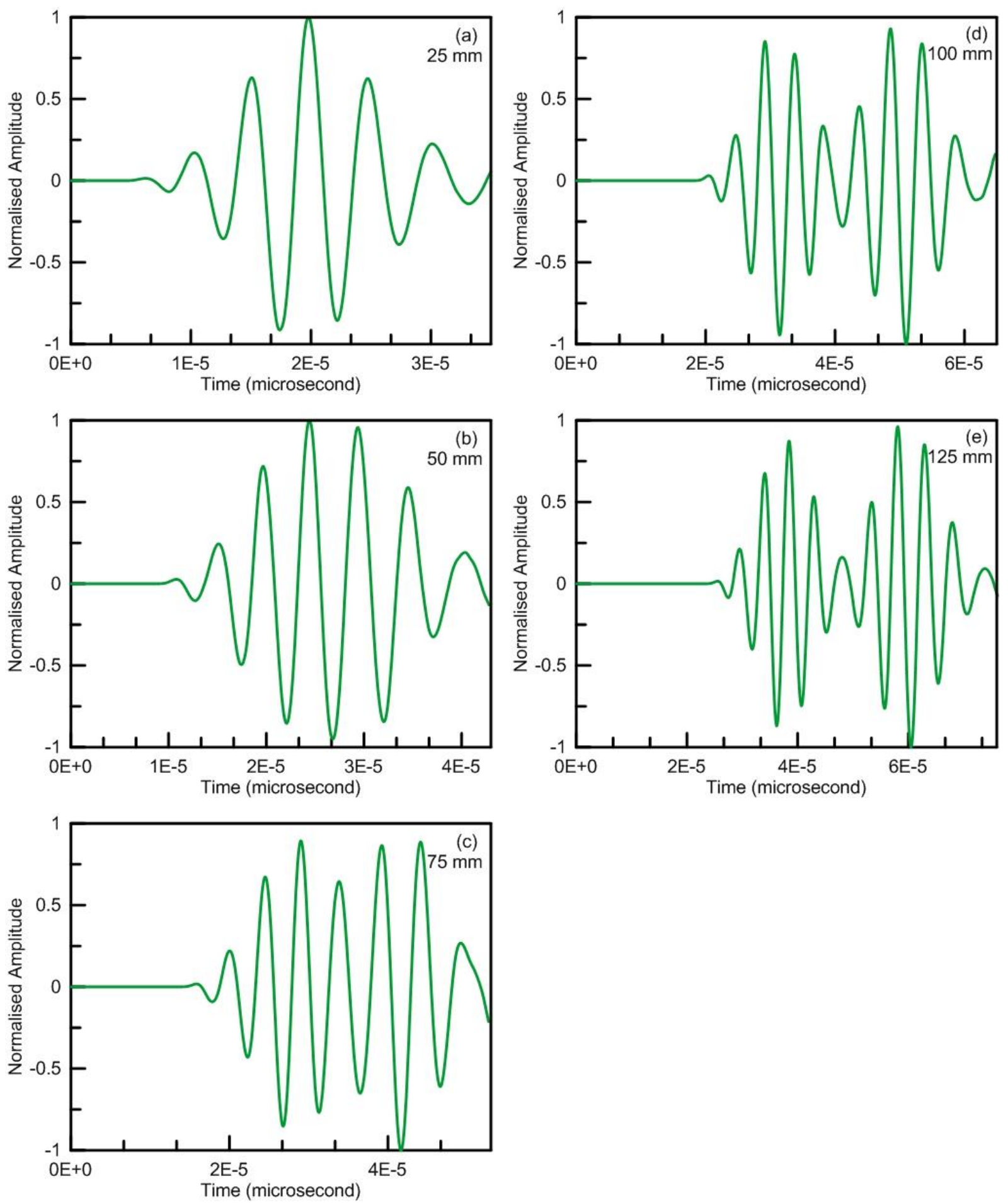

Figure 8: Guided-wave received at the sensor calculated by the numerical solution for the distances between the actuator and sensor of: (a) $25 \mathrm{~mm}$, (b) $50 \mathrm{~mm}$, (c) $75 \mathrm{~mm}$, (d) $100 \mathrm{~mm}$ and (e) $125 \mathrm{~mm}$. 

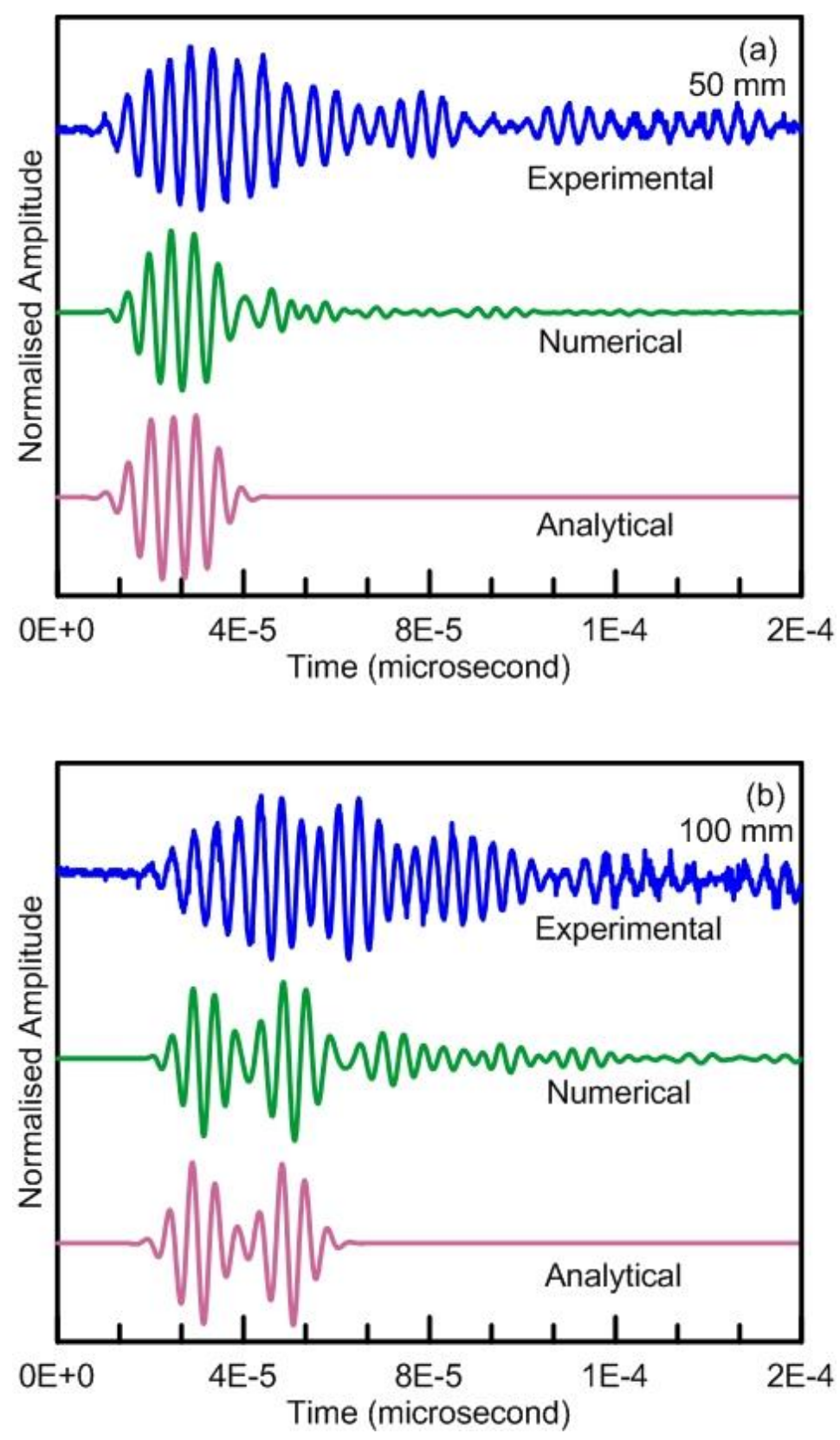

Figure 9: Comparison of the analytical, numerical and experimental results for the distances between the both transducers (a) 50mm and (b) $100 \mathrm{~mm}$. 


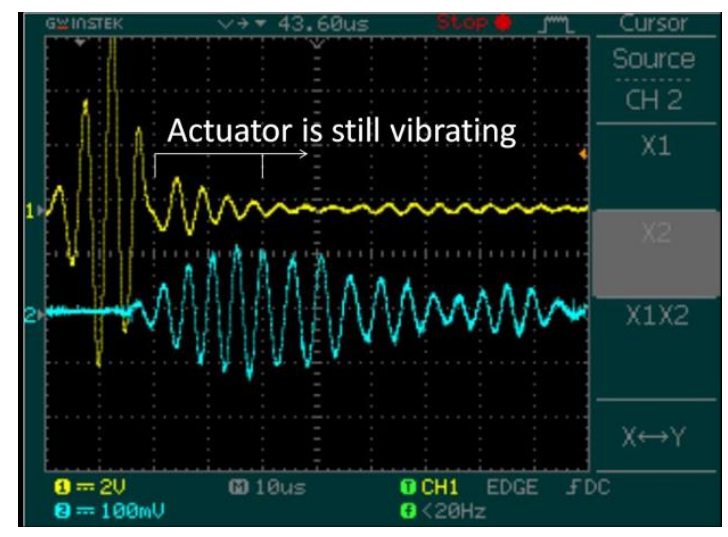

Figure 10: Screen shot of the oscilloscope showing signals from the actuator (above) and received signals at the sensor (below). 
2016-11-28

Response of Gaussian-modulated guided wave in aluminum: An analytical, numerical, and experimental study

Khan, Sohaib Z.

SAGE Publications (UK and US)

Khan SZ, Khan MA, Tariq M., Response of Gaussian-modulated guided wave in aluminum: An analytical, numerical, and experimental study, Proceedings of the Institution of Mechanical Engineers, Part C: Journal of Mechanical Engineering Science, Volume 231, Issue 16, 2017 , pp. 3057-3065

http://dx.doi.org/10.1177/0954406216681594

Downloaded from Cranfield Library Services E-Repository 